\title{
Massive Vector Fields in Rotating Black-Hole Spacetimes: Separability and Quasinormal Modes
}

\author{
Valeri P. Frolov, ${ }^{1, *}$ Pavel Krtouš, ${ }^{2, \dagger}$ David Kubizňák, ${ }^{3, *}$ and Jorge E. Santos ${ }^{4, \S}$ \\ ${ }^{1}$ Theoretical Physics Institute, University of Alberta, Edmonton, Alberta, Canada T6G $2 E 1$ \\ ${ }^{2}$ Institute of Theoretical Physics, Faculty of Mathematics and Physics, Charles University, \\ V Holešovičkách 2, Prague 18000, Czech Republic \\ ${ }^{3}$ Perimeter Institute, 31 Caroline Street North, Waterloo, Ontario N2L 2Y5, Canada \\ ${ }^{4}$ DAMTP, Centre for Mathematical Sciences, Wilberforce Road, Cambridge CB3 OWA, United Kingdom
}

(Received 9 April 2018; published 6 June 2018)

\begin{abstract}
We demonstrate the separability of the massive vector (Proca) field equation in general Kerr-NUT-AdS black-hole spacetimes in any number of dimensions, filling a long-standing gap in the literature. The obtained separated equations are studied in more detail for the four-dimensional Kerr geometry and the corresponding quasinormal modes are calculated. Two of the three independent polarizations of the Proca field are shown to emerge from the separation ansatz and the results are found in an excellent agreement with those of the recent numerical study where the full coupled partial differential equations were tackled without using the separability property.
\end{abstract}

DOI: 10.1103/PhysRevLett.120.231103

Introduction.-Maxwell equations describe a theory of a massless vector field (photons). When the massive term is added, a theory of the massive spin-1 vector particle is obtained. The massive term breaks the gauge invariance. Historically, the massive vector mesons were first used by Proca [1] in his attempt to explain the short-range nuclear forces (see also [2,3]). In modern physics, the Proca equation is used in the standard model for describing the massive spin-1 $Z$ and $W$ bosons. The Proca equation, originally formulated in a flat spacetime, can easily be generalized to the curved background, see, e.g., [4]. Recently, the interest in the Proca fields greatly increased after it was demonstrated that ultralight vector fields might be responsible for instability of rotating black holes.

The ultralight massive vector fields, such as dark photons, are a feature of many beyond standard model physics scenarios, string theory for example [5]. Together with axionlike scalars such fields provide a compelling candidate for cold dark matter [6]. However, due to their tiny mass and weak coupling, direct searches for such particles are challenging, and alternative methods for detection are sought. One such promising method is to look for the superradiant instabilities of rotating black holes, leading to a formation of the bosonic condensate outside the horizon, and a subsequent gravitational wave

Published by the American Physical Society under the terms of the Creative Commons Attribution 4.0 International license. Further distribution of this work must maintain attribution to the author(s) and the published article's title, journal citation, and DOI. Funded by SCOAP . emission and spin-down of the black hole. Consequently, gravitational and electromagnetic wave observations of astrophysical black holes provide direct constraints on certain models of ultralight massive particles, e.g., [7-9].

In order to make these predictions, one has to (in the first approximation) study the behavior of the corresponding test fields in the background of the rotating black hole. For the massive vector field this is a formidable task, as the corresponding equations were believed not to separate [10-12] and the problem was studied either using certain approximations, e.g., $[8,10,12,13]$, or by applying heavy numerics $[9,14,15]$.

In this Letter, we show that no such limitations are necessary. Namely, we demonstrate that with a proper ansatz the massive vector field equation does separate and that this not only happens for the four-dimensional vacuum Kerr spacetime, but remains also true in higher dimensions and in the presence of the cosmological constant and NUT charges. Not only this result fills a long-standing gap in the literature on separability of physical field equations in the black-hole spacetimes, e.g., [16], but it also provides an opportunity to study the properties of these fields (for example their superradiant instability) in a significantly simplified manner.

In this Letter we proceed as follows. We first demonstrate separability of the massive vector field equations in the Kerr-NUT-AdS black-hole spacetimes in any number of dimensions [17] and relate this separability to the existence of the hidden symmetry present in these spacetimes [16]. This result is new and nontrivial even in four dimensionsfor the Kerr geometry. Next, restricting to this case, we demonstrate that our ansatz correctly captures two of the 
three independent polarizations of the Proca field and that we are able to much more effectively reconstruct the unstable modes recently studied in [9] by heavy numerical methods. We conclude with some general remarks on possible future directions.

Spacetime geometry.-In what follows we want to demonstrate the separability of the four- and higherdimensional Proca equations in the background of a wide class of metrics that include the general Kerr-NUT-AdS solutions [17] as a special case. Such a separability is valid in both even and odd dimensions; however, the forms of the metric and of the separated equations are slightly different. For brevity, in this Letter we restrict to the case of even dimensions, $D=2 N$.

The metrics we consider are of the form

$$
\boldsymbol{g}=\sum_{\mu=1}^{N}\left[\frac{U_{\mu}}{X_{\mu}} \boldsymbol{d} x_{\mu}^{2}+\frac{X_{\mu}}{U_{\mu}}\left(\sum_{j=0}^{N-1} A_{\mu}^{(j)} \boldsymbol{d} \psi_{j}\right)^{2}\right] .
$$

Here, $A_{\mu}^{(j)}$ and $U_{\mu}$ are polynomials in $x_{\mu}^{2}$ defined by the following relations:

$$
\begin{gathered}
A_{\mu}(\beta) \equiv \prod_{\substack{\nu=1 \\
\nu \neq \mu}}^{N}\left(1+\beta^{2} x_{\nu}^{2}\right)=\sum_{j=0}^{N-1} A_{\mu}^{(j)} \beta^{2 j}, \\
U_{\mu}=\prod_{\substack{\nu=1 \\
\nu \neq \mu}}^{N}\left(x_{\nu}^{2}-x_{\mu}^{2}\right),
\end{gathered}
$$

and $X_{\mu}$ are arbitrary functions of one variable, $X_{\mu}\left(x_{\mu}\right)$, cf. [16]. We call the metric (1) an off-shell metric, to distinguish it from a special case when (1) obeys the Einstein equations with or without the cosmological constant. In such a case functions $X_{\mu}$ become special polynomials and the metric describes the Kerr-NUT-AdS solution [17].

The characteristic property of the metric (1) is that it admits the principal tensor $\boldsymbol{h}$, see [16]. The principal tensor is a nondegenerate closed conformal Killing-Yano 2-form. It satisfies the equation

$$
\nabla_{c} h_{a b}=g_{c a} \xi_{b}-g_{c b} \xi_{a},
$$

where $\xi$ is a primary Killing vector,

$$
\xi_{a}=\frac{1}{D-1} \nabla^{b} h_{b a} .
$$

The off-shell metric possesses $N$ Killing vectors

$$
\boldsymbol{l}_{(j)}=\partial_{\psi_{j}}, \quad j=0, \ldots, N-1,
$$

the first of which coincides with the primary Killing vector, $\boldsymbol{l}_{(0)}=\boldsymbol{\xi}$. The remaining $N$ canonical coordinates, $x_{\mu}$, $\mu=1, \ldots, N$, are nothing but the eigenvalues of the principal tensor $\boldsymbol{h}$, which in the canonical coordinates $\left(x_{\mu}, \psi_{j}\right)$ takes the following form:

$$
\boldsymbol{h}=\sum_{\mu=1}^{N} x_{\mu} \boldsymbol{d} x_{\mu} \wedge\left(\sum_{j=0}^{N-1} A_{\mu}^{(j)} \boldsymbol{d} \psi_{j}\right)
$$

Separation of variables.-Our goal is to demonstrate the separability of the Proca equation

$$
\nabla_{n} \mathrm{~F}^{a n}+m^{2} \mathrm{~A}^{a}=0
$$

in the metric (1). Here $\mathrm{F}_{a n}=\nabla_{a} \mathrm{~A}_{n}-\nabla_{n} \mathrm{~A}_{a}$, and $m$ is the mass of the massive vector field $\mathbf{A}$. To this purpose we shall use the following ansatz [18-20]:

$$
\mathrm{A}^{a}=B^{a b} \nabla_{b} Z, \quad B^{a b}\left(g_{b c}+i \mu h_{b c}\right)=\delta_{c}^{a} .
$$

Here, $\mu$ is a complex parameter, and the potential function $Z$ is written in the multiplicative separated form

$$
Z=\prod_{\nu=1}^{N} R_{\nu}\left(x_{\nu}\right) \exp \left(i \sum_{j=0}^{N-1} L_{j} \psi_{j}\right)
$$

An immediate consequence of the Proca equation is the "Lorenz condition,"

$$
\nabla_{a} \mathrm{~A}^{a}=0 .
$$

As shown in [20], for the ansatz (9), this explicitly reads

$$
\nabla_{a} \mathrm{~A}^{a}=\frac{Z}{A} \sum_{\nu=1}^{N} \frac{A_{\nu}}{U_{\nu}} \frac{1}{R_{\nu}} \mathcal{D}_{\nu} \mathcal{R}_{\nu}
$$

where

$$
\begin{aligned}
\mathcal{D}_{\nu}= & q_{\nu} \frac{\partial}{\partial x_{\nu}}\left[\frac{X_{\nu}}{q_{\nu}} \frac{\partial}{\partial x_{\nu}}\right]-\frac{1}{X_{\nu}}\left[\sum_{j=0}^{N-1}\left(-x_{\nu}^{2}\right)^{N-1-j} L_{j}\right]^{2} \\
& +\mu \frac{2-q_{\nu}}{q_{\nu}}\left(-\mu^{2}\right)^{(1-N)} \sum_{j=0}^{N-1}\left(-\mu^{2}\right)^{j} L_{j},
\end{aligned}
$$

and

$$
q_{\nu}=1-\mu^{2} x_{\nu}^{2}, \quad A=\prod_{\nu=1}^{N} q_{\nu}
$$

The Lorenz condition (11) may be satisfied provided the mode functions $R_{\nu}$ obey the separated equations

$$
\mathcal{D}_{\nu} R_{\nu}=\tilde{C}_{\nu} R_{\nu},
$$


where $\tilde{C}_{\nu}$ are polynomials in variable $x_{\nu}^{2}$ with the same coefficients $C_{k}$,

$$
\tilde{C}_{\nu}=\sum_{k=0}^{N-1} C_{k}\left(-x_{\nu}^{2}\right)^{N-1-k}
$$

Indeed, in such a case the expression in (12) sums to

$$
\nabla_{a} \mathrm{~A}^{a}=\frac{Z}{A} \sum_{j=0}^{N-1} C_{j}\left(-\mu^{2}\right)^{j},
$$

and we see that the Lorenz condition holds provided the parameter $\mu$ satisfies the following constraint:

$$
\sum_{j=0}^{N-1} C_{j}\left(-\mu^{2}\right)^{j}=0 .
$$

The results of [20] can be also used to find the representation of $\nabla_{n} \mathrm{~F}^{a n}$ for the ansatz (9). When the Lorenz condition (11) holds, one has

$$
\nabla_{n} \mathrm{~F}^{a n}+m^{2} \mathrm{~A}^{a}=-B^{a m} \nabla_{m} J,
$$

with

$$
J=\square Z+2 \beta \xi_{k} B^{k n} \nabla_{n} Z-m^{2} Z .
$$

The key observation is that $J$ also separates in the form

$$
J=Z \sum_{\nu=1}^{N} \frac{1}{U_{\nu}} \frac{1}{R_{\nu}}\left[\mathcal{D}_{\nu}-m^{2}\left(-x_{\nu}^{2}\right)^{N-1}\right] R_{\nu},
$$

where the mass term has been rewritten using the identity:

$$
\sum_{\nu=1}^{N} \frac{1}{U_{\nu}}\left(-x_{\nu}^{2}\right)^{N-1-j}=\delta_{0}^{j}
$$

for $j=0$. The same identity guarantees $J=0$, provided the modes $R_{\nu}\left(x_{\nu}\right)$ obey separated equations (15) with an additional condition that the coefficient $C_{0}$ of the highest order term in the polynomials $\tilde{C}_{\nu}$ is given by the mass,

$$
C_{0}=m^{2} .
$$

This finishes the proof of separability of the Proca equation in the off shell Kerr-NUT-AdS metrics.

Summarizing, the Proca equation (8) for the vector field A in the form (9) can be solved using the multiplicative separation ansatz (10), where the mode functions $R_{\nu}$ satisfy ordinary differential equations (ODEs) (15) with separation constants $C_{k}$ and $L_{j}$, provided that $C_{0}$ is given by (23) and $\mu$ satisfies (18). The obtained mode functions are thus labeled by the full set of $2 N-1=D-1$ separation constants, and in that sense, they are completely general. However, at the moment it is not clear how the polarizations of the field are captured by our ansatz. One can speculate that the choice of the root $\mu$ in the constraint (18) could be related to the choice of polarization. If so, the obtained solutions describe $N-1$ complex polarization modes, that is, $D-2$ real modes, and only one polarization is missing. This speculation is supported by the numerics described below. However, further discussion of this issue is necessary.

Quasinormal modes.-The demonstrated separability of the Proca equation has many interesting applications. For example, it allows one to study the problem of the stability of rotating black holes with respect to the massive vector field condensation. This problem reduces to a study of the spectrum of separated ODEs and the corresponding calculation of the complex frequency of nonradiative quasinormal modes (sometimes called quasibound states or unstable modes). For original (nonseparated) equations, the required numerical calculations are very time consuming, as one has to solve a system of partial differential equations (PDEs). The study of quasinormal modes by using the separated Proca equation has many advantages.

In order to illustrate this, we focus on the case of fourdimensional Kerr spacetime $(N=2)$ studied recently in [9], setting both the NUT parameter and the cosmological constant equal to zero. We will show that our separation ansatz correctly reproduces two of the three physical polarizations of the massive vector in this spacetime. The polarizations captured by our analysis are labeled by $S= \pm 1$, and they reduce to those explored in [11] when the black-hole spin vanishes (see also $[21,22]$ ).

Before proceeding, let us translate our results to a more familiar Boyer-Lindquist frame. First, we change from the canonical coordinates $\left(\psi_{0}, x_{1}, x_{2}, \psi_{1}\right)$ to new coordinates $(t, r, \theta, \phi)$ via the map

$$
\left(\psi_{0}, x_{1}, x_{2}, \psi_{1}\right)=(t-a \phi, i r, a \cos \theta, \phi / a),
$$

and identify

$X_{1}=2 M r-r^{2}-a^{2} \equiv-\Delta, \quad X_{2}=-a^{2} \sin ^{2} \theta$.

Upon this, the metric element (1) yields the standard Boyer-Lindquist form of the Kerr geometry. The blackhole horizon is a null hypersurface located at $r=r_{+} \equiv$ $M+\sqrt{M^{2}-a^{2}}$; we are interested in the subextremal limit for which $M<|a|$. Finally, we want to map the eigenvalues $L_{0}$ and $L_{1}$, to the eigenvalues of $i \partial_{t}$ and $-i \partial_{\phi}, \omega$ and $m_{\phi}$. This is accomplished via the linear map

$$
L_{0}=-\omega, \quad L_{1}=a\left(m_{\phi}-a \omega\right) .
$$

Note that, since $\phi$ has period $2 \pi$, we have $m_{\phi} \in \mathbb{Z}$. 
Equations (15) reduce to two differential equations in $r$ and $\theta$, respectively, which only couple to each other via their dependence on the parameters $\left\{\mu, \omega, m_{\phi}, m, M, a\right\}$ :

$$
\frac{d}{d r}\left[\frac{\Delta}{q_{r}} \frac{d R}{d r}\right]+\left[\frac{K_{r}^{2}}{q_{r} \Delta}+\frac{2-q_{r}}{q_{r}^{2}} \frac{\sigma}{\mu}-\frac{m^{2}}{\mu^{2}}\right] R=0,
$$

$\frac{1}{\sin \theta} \frac{d}{d \theta}\left[\frac{\sin \theta}{q_{\theta}} \frac{d S}{d \theta}\right]-\left[\frac{K_{\theta}^{2}}{q_{\theta} \sin ^{2} \theta}+\frac{2-q_{\theta}}{q_{\theta}^{2}} \frac{\sigma}{\mu}-\frac{m^{2}}{\mu^{2}}\right] S=0$,

where

$$
\begin{aligned}
K_{r} & =a m_{\phi}-\left(a^{2}+r^{2}\right) \omega, & K_{\theta} & =m_{\phi}-a \omega \sin ^{2} \theta, \\
q_{r} & =1+\mu^{2} r^{2}, & q_{\theta} & =1-\mu^{2} a^{2} \cos ^{2} \theta, \\
\sigma & =a \mu^{2}\left(m_{\phi}-a \omega\right)+\omega . & &
\end{aligned}
$$

Note that, at this stage, we have set

$$
C_{0}=m^{2}, \quad C_{1}=m^{2} / \mu^{2},
$$

so the constraints (18) and (23) are readily solved.

Equations (27) are then solved as a coupled eigenvalue problem, with an eigenvalue pair $(\mu, \omega)$. The numerical method follows mutatis mutandis [9], and it will not be detailed here again. The boundary conditions are chosen so that the modes are regular on the black-hole horizon and have finite energy. In Fig. 1, we show the results of determining the lowest lying quasinormal mode for $a / M=0.998$, and $m_{\phi}=1$. The (blue) circles represent our numerical data, and the (orange) squares the numerical data of [9]. The values agree with each other to within $10^{-8} \%$.

The advantage of solving (27) instead of the coupled PDE system in [9] is immense. For instance, we were able to extend the polar mode of [9] with $S=-1$ and $m_{\phi}=1$, which is the most unstable mode, to masses as small as $m M \sim 10^{-3}$. In [8], the quasinormal mode spectrum of this mode was computed in the small mass regime $\tilde{m} \equiv m M \ll 1$, and it was found to be given by

$$
M \omega \approx \tilde{m}\left(1-\frac{\tilde{m}^{2}}{2}\right)+2 i\left(\frac{a}{M}-2 \tilde{m}\right) \tilde{m}^{7}
$$

When $m M \ll 1$, the PDEs of [9] become very hard to solve, so the regime of validity of this approximation was never identified. Here, we close this gap in the literature and compare our exact numerical results in Fig. 1, depicted as (blue) circles, to the analytic approximation (30) represented as the dashed black line. When $m M \ll 1$, the agreement is excellent. Additionally, to modes calculated in [9], we have also computed the lowest lying quasinormal mode with $S=+1$, which is depicted as the bottom (green) curve of Fig. 1. Our field ansatz thus covers two polarization modes - these reduce to the even-parity (polar) scalar modes in the nonrotating limit. The scaling at small $m M$ of both polarizations is consistent with the one reported in $[8,10-12]$. The question of how to recover the last (axial) polarization remains currently open.

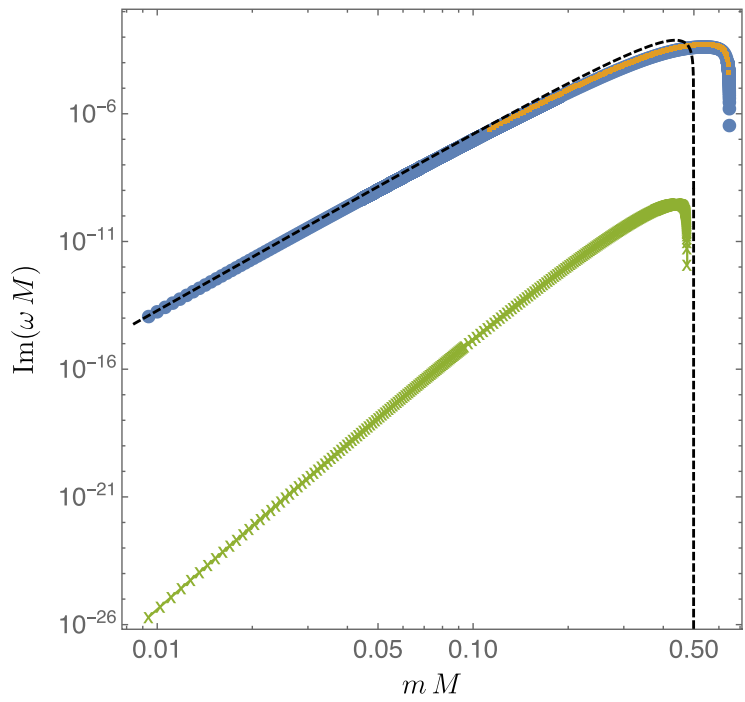

FIG. 1. Quasinormal modes. We display the frequency $\omega M$ as a function of $m M$ computed for quasinormal modes with $m_{\phi}=1$ and $a / M=0.998$. The real (left) and imaginary (right) part of $\omega M$ is displayed for the two polarizations $S=-1$, +1 , with the top (online blue) curve corresponding to $S=-1$, and the lower (online green) one to $S=+1$. The polarization $S=-1$ corresponds to the most unstable mode. For this case, the (online orange) squares represent the data from [9], and the dashed line gives the analytic approximation (30) derived in [8]. Solving separated ODEs allowed us to extend the numerics below $m M \sim 10^{-2}$, compared to the range $m M \gtrsim 10^{-1}$ of [9]. The agreement of the analytic approximation with our numerics for small $m M$ is excellent. 
Conclusions.-We have shown that the Proca field equation in a general Kerr-NUT-AdS spacetime separates in all dimensions. The key ingredient for this result is the ansatz (9). It is written purely in terms of the principal tensor, and thus, it encodes explicit and hidden symmetries of the spacetime.

Furthermore, we have used the separated equation to study the quasinormal mode spectrum of the Proca field around the Kerr black hole. Our spectrum matches that of [9] for two of the three polarizations. Except, solving just ODEs allows much more effective calculations.

The numerics confirm that our method captures more than one polarization. On general grounds, we have speculated that only one polarization mode is missing. However, the question as to how precisely these polarizations are described by the ansatz (9) and how to obtain the remaining polarization(s) remains open.

Our results open a myriad of opportunities for the near future. At a more mathematical level, we could hope to use the results of [23] and prove the existence of exponentially growing modes for Proca perturbations around the Kerr black hole. Although both our work and [9] give ample numerical evidence that this is the case, it would be reassuring to have a definite mathematical proof. Also interesting, would be to repeat Detweiler's seminal calculation [24], and then analytically extract the growth rate of Proca fields for $m M, a M \ll 1$. Finally, we would like to mention that, still in four dimensions, we could study the stability of the Proca field for $\Lambda<0$, with the latter scenario being relevant in the context of the AdS/CFT correspondence. Perhaps more interestingly, virtually nothing is known about Proca perturbations in the context of higher-dimensional black holes. Our separation ansatz can be equally used in those circumstances.

V.F. thanks the Natural Sciences and Engineering Research Council of Canada (NSERC) and the Killam Trust for their financial support. P. K. is supported by Czech Science Foundation Grant No. 17-01625S. D. K. acknowledges the Perimeter Institute for Theoretical Physics and the NSERC for their support. Research at Perimeter Institute is supported by the Government of Canada through the Department of Innovation, Science and Economic Development Canada and by the Province of Ontario through the Ministry of Research, Innovation and Science. J.E. S. is supported in part by STFC Grants No. PHY-1504541 and No. ST/P000681/1.

\footnotetext{
vfrolov@ualberta.ca

†Pavel.Krtous@utf.mff.cuni.cz

\$dkubiznak@perimeterinstitute.ca

§js555@cam.ac.uk
}

[1] A. Proca, Sur la théorie ondulatoire des électrons positifs et négatifs, J. Phys. Radium 7, 347 (1936).
[2] F. J. Belinfante, The interaction representation of the Proca field, Phys. Rev. 76, 66 (1949).

[3] N Rosen, A classical Proca particle, Found. Phys. 24, 1689 (1994).

[4] M. Seitz, Proca field in a space-time with curvature and torsion, Classical Quantum Gravity 3, 1265 (1986).

[5] M. Goodsell, J. Jaeckel, J. Redondo, and A. Ringwald, Naturally light hidden photons in LARGE volume string compactifications, J. High Energy Phys. 11 (2009) 027.

[6] R. Essig et al., Working group report: New light weakly coupled particles, in Proceedings, 2013 Community Summer Study on the Future of U.S. Particle Physics: Snowmass on the Mississippi (CSS2013): Minneapolis, MN, USA, July 29-August 6, 2013.

[7] A. Arvanitaki, S. Dimopoulos, S. Dubovsky, N. Kaloper, and J. March-Russell, String axiverse, Phys. Rev. D 81, 123530 (2010).

[8] M. Baryakhtar, R. Lasenby, and M. Teo, Black hole superradiance signatures of ultralight vectors, Phys. Rev. D 96, 035019 (2017).

[9] V. Cardoso, O. J. C. Dias, G. S. Hartnett, M. Middleton, P. Pani, and J.E. Santos, Constraining the mass of dark photons and axion-like particles through black-hole superradiance, J. Cosmol. Astropart. Phys. 03 (2018) 043.

[10] P. Pani, V. Cardoso, L. Gualtieri, E. Berti, and A. Ishibashi, Black Hole Bombs and Photon Mass Bounds, Phys. Rev. Lett. 109, 131102 (2012).

[11] J. G. Rosa and S. R. Dolan, Massive vector fields on the Schwarzschild spacetime: quasinormal modes and bound states, Phys. Rev. D 85, 044043 (2012).

[12] P. Pani, V. Cardoso, L. Gualtieri, E. Berti, and A. Ishibashi, Perturbations of slowly rotating black holes: massive vector fields in the Kerr metric, Phys. Rev. D 86, 104017 (2012).

[13] S. Endlich and R. Penco, A modern approach to superradiance, J. High Energy Phys. 05 (2017) 052.

[14] W. E. East and F. Pretorius, Superradiant Instability and Backreaction of Massive Vector Fields Around Kerr Black Holes, Phys. Rev. Lett. 119, 041101 (2017).

[15] W. E. East, Superradiant instability of massive vector fields around spinning black holes in the relativistic regime, Phys. Rev. D 96, 024004 (2017).

[16] V. P. Frolov, P. Krtouš, and D. Kubizňák, Black holes, hidden symmetries, and complete integrability, Living Rev. Relativity 20, 6 (2017).

[17] W. Chen, H. Lu, and C. N. Pope, General Kerr-NUT-AdS metrics in all dimensions, Classical Quantum Gravity 23, 5323 (2006).

[18] O. Lunin, Maxwell's equations in the Myers-Perry geometry, J. High Energy Phys. 12 (2017) 138.

[19] V. P. Frolov, P. Krtouš, and D. Kubizňák, Separation variables in Maxwell equations in Plebański-Demiański metric, Phys. Rev. D 97, 101701(R) (2018).

[20] P. Krtouš, V. P. Frolov, and D. Kubizňák, Separation of Maxwell equations in Kerr-NUT-(A)dS spacetimes, arXiv: 1803.02485 .

[21] R. A. Konoplya, A. Zhidenko, and C. Molina, Late time tails of the massive vector field in a black hole background, Phys. Rev. D 75, 084004 (2007). 
[22] R. A. Konoplya, Massive vector field perturbations in the Schwarzschild background: Stability and unusual quasinormal spectrum, Phys. Rev. D 73, 024009 (2006).

[23] Y. Shlapentokh-Rothman, Exponentially growing finite energy solutions for the Klein-Gordon equation on sub-extremal Kerr spacetimes, Commun. Math. Phys. 329, 859 (2014).

[24] S. L. Detweiler, Klein-Gordon equation and rotating black holes, Phys. Rev. D 22, 2323 (1980). 